\title{
Establishing neuronal identity in vertebrate neurogenic placodes
}

\author{
Clare V. H. Baker* and Marianne Bronner-Fraser \\ Division of Biology, Beckman Institute 139-74, California Institute of Technology, Pasadena, California 91125, USA \\ *Author for correspondence (e-mail: cbaker@caltech.edu)
}

Accepted 28 April; published on WWW 22 June 2000

\section{SUMMARY}

The trigeminal and epibranchial placodes of vertebrate embryos form different types of sensory neurons. The trigeminal placodes form cutaneous sensory neurons that innervate the face and jaws, while the epibranchial placodes (geniculate, petrosal and nodose) form visceral sensory neurons that innervate taste buds and visceral organs. In the chick embryo, the ophthalmic trigeminal (opV) placode expresses the paired homeodomain transcription factor Pax3 from very early stages, while the epibranchial placodes express Pax2. Here, we show that Pax3 expression in explanted opV placode ectoderm correlates at the single cell level with neuronal specification and with commitment to an opV fate. When opV (trigeminal) ectoderm is grafted in place of the nodose (epibranchial) placode, Pax3-expressing cells form Pax3positive neurons on the same schedule as in the opV placode. In contrast, Pax3-negative cells in the grafted ectoderm are induced to express the epibranchial placode marker Pax2 and form neurons in the nodose ganglion that express the epibranchial neuron marker Phox $2 a$ on the same schedule as host nodose neurons. They also project neurites along central and peripheral nodose neurite pathways and survive until well after the main period of cell death in the nodose ganglion. The older the opV ectoderm is at the time of grafting, the more Pax3-positive cells it contains and the more committed it is to an opV fate. Our results suggest that, within the neurogenic placodes, there does not appear to be a two-step induction of 'generic' neurons followed by specification of the neuron to a particular fate. Instead, there seems to be a one-step induction in which neuronal subtype identity is coupled to neuronal differentiation.

Key words: Placodes, Trigeminal placode, nodose placode, Pax2, Pax3, Phox2a, Chick, Quail, Sensory neurons

\section{INTRODUCTION}

Placodes are discrete regions of thickened columnar epithelium that form in characteristic positions in the head of vertebrate embryos (Le Douarin et al., 1986; Webb and Noden, 1993). They make vital contributions to the paired sense organs (eyes, ears and nose) and to the peripheral sensory nervous system in the head. The trigeminal and epibranchial (geniculate, petrosal and nodose) placodes all form sensory neurons, but these neurons perform different functions according to their placode of origin. For example, the two trigeminal placodes (ophthalmic and maxillomandibular) form cutaneous sensory neurons in the trigeminal ganglion that transmit touch, pain and temperature information from the face and jaws. The nodose placode produces visceral sensory neurons in the distal ganglion of the vagal nerve that innervate a variety of receptors in the heart, lungs and gastrointestinal tract. Nodose neurons transmit information about, for example, heart rate, blood pressure, bronchial irritation and visceral distension. Much is known about the growth and survival factors required throughout development by neurons in the different placodederived ganglia (reviewed by Lindsay, 1996; Davies, 1998). However, the mechanism by which different types of neurons are generated from different placodes is poorly understood.

Placode induction is a lengthy process that begins in late gastrula stages, involving a series of inducers from different embryonic tissues including endoderm, mesoderm and the neural tube (Jacobson, 1963a,b,c). Historically, the commitment of placodal ectoderm towards a specific placodal fate has been tested either by substituting different placodal primordia for one another, or by grafting them into nonplacode-forming locations like the trunk. Substitution experiments have identified successive stages in placodal commitment. Grafts performed at very early stages can lead to adoption of a fate entirely appropriate to the graft location, suggesting that commitment towards a particular placodal fate has not yet occurred (Vogel and Davies, 1993). By tailbud stages, most placodes are no longer able to change their function when grafted to ectopic positions (Stone, 1924, 1928, 1929).

It is possible to look more precisely at different stages in placode development by determining when placodal ectoderm is committed to express a specific early molecular marker in ectopic environments. Such experiments address the plasticity of placodal ectoderm at different times in development, and the extent to which early marker expression correlates with the commitment of the ectoderm to a specific placodal fate. In recent years, members of the Pax family of paired domain transcription factors (reviewed by Mansouri et al., 1996; Dahl et al., 1997) have been found to be expressed from early stages 
in different placodes, suggesting the existence of a placodal 'Pax code'. Pax6 is essential for the development of the lens and olfactory placodes (Hill et al., 1991; Hogan et al., 1986), while Pax2 is essential for the development of the otic placode (Favor et al., 1996; Torres et al., 1996). We recently showed that $\operatorname{Pax} 3$ is an early molecular marker for the ophthalmic trigeminal $(\mathrm{opV})$ placode and performed a detailed analysis of its induction (Baker et al., 1999; Stark et al., 1997).

Here, we show that Pax3 expression in explanted opV ectoderm correlates with neuronal specification and with commitment to an opV fate. When opV ectoderm is grafted to the nodose placode region, Pax3-expressing cells form Pax3positive neurons that differentiate on the same schedule as opV neurons. Non-Pax3-expressing cells are induced to express the epibranchial placode marker Pax2 and form neurons in the nodose ganglion on the same schedule as nodose neurons. These neurons express the epibranchial placode-specific marker Phox $2 a$, project along nodose-specific central and peripheral pathways, and survive until well after the main period of cell death in the nodose ganglion. As the number of Pax3-expressing cells increases, competence to express epibranchial placode markers decreases. Hence, within the placodes, there does not appear to be a two-step induction of 'generic' neurons followed by specification of the neurons to a particular fate. Instead, neuronal subtype identity seems to be coupled to neuronal differentiation in a one-step induction process.

\section{MATERIALS AND METHODS}

\section{Quail-chick grafts}

Quail and chick eggs were prepared and grafts performed as previously described (Baker et al., 1999). For some grafting experiments, the donor quail embryos were dispase-treated before isolation of the ectoderm. Embryos were explanted into cold Ringer's solution and incubated in $1 \mathrm{mg} / \mathrm{ml}$ dispase in Hepes-buffered DMEM for 15 minutes on ice and 10 minutes at $37^{\circ} \mathrm{C}$. After at least 10 minutes recovery on ice in complete medium (10\% horse serum, $15 \%$ chick embryo extract in Eagle's Minimal Essential Medium), they were transferred to cold Ringer's solution for dissection. When grafting dispase-treated ectoderm, a drop of ice-cold Ringer's solution was placed on the host embryo as this helped to reduce curling of the grafted ectoderm.

\section{Surface Dil labelling}

DiI labelling of surface ectoderm was performed on 8-somite stage and older embryos so that placode-derived cells could be distinguished from underlying neural crest cells during analysis (Stark et al., 1997). Briefly, $50 \mu \mathrm{g}$ CellTracker DiI (Molecular Probes) were resuspended in $25 \mu \mathrm{l}$ ethanol and then diluted to $400 \mu \mathrm{l}$ with $10 \%$ sucrose. The vitelline membrane was removed from over the midbrain region and a few drops of DiI mouth-pipetted onto the surface of the embryo. After 10-20 minutes, the embryos were washed extensively in Ringer's solution and the ectoderm removed using pulled glass needles.

\section{Collagen gel cultures}

Collagen matrix gels were prepared as previously described (Baker et al., 1999) and cultured for 20-36 hours in F-12 medium plus N-2 supplements (Gibco-BRL) at $38^{\circ} \mathrm{C}, 5 \% \mathrm{CO}_{2}$. They were fixed in $4 \%$ paraformaldehyde for 1-2 hours, and processed for cryostat sectioning as described by Sechrist et al. (1995).

\section{Immunohistochemistry}

Embryos collected after 0 hours to 2 days incubation were fixed in $4 \%$ paraformaldehyde for 1-2 hours and processed for cryostat sectioning as described by Sechrist et al. (1995). Embryos collected after 4-5 days incubation were fixed overnight in $4 \%$ paraformaldehyde at $4{ }^{\circ} \mathrm{C}$ and similarly processed. $12 \mu \mathrm{m}$ sections of embryos or collagen gels (see above) were mounted on Superfrost ${ }^{\circledR}$ Plus glass slides (Fisher) and the gelatin removed by treating the slides in phosphate-buffered saline (PBS) at $42^{\circ} \mathrm{C}$ for 5 minutes. Antibodies (see below) were diluted in PBS containing $0.1 \%$ bovine serum albumin and $0.1 \%$ Triton X-100. For the anti-quail neurite QN antibody only, slides were blocked for 1 hour in $10 \%$ fetal bovine serum in the same solution. Slides were generally incubated overnight at $4{ }^{\circ} \mathrm{C}$ in primary antibody solution, and for $1-2$ hours in secondary antibody solution, with three 5-minute washes in PBS between each step. Where biotinylated secondary antibodies were used, slides were further incubated for 1 hour in avidin-NeutrAvidin ${ }^{\mathrm{TM}}$ Alexa $^{\mathrm{TM}} 350$ (Molecular Probes) at 1:100 in PBS, followed by three 5-minute washes in PBS. Slides were washed in distilled water, mounted in Fluoromount-G (Southern Biotechnology) and viewed by fluorescence microscopy. Only those chimeras that had successfully incorporated the donor graft were scored.

Primary antibodies were used as follows: 1:1 anti-quail QCPN supernatant (mouse IgG1; Developmental Studies Hybridoma Bank, University of Iowa); 1:5000 anti-Pax3 ascites (mouse IgG2a; see Baker et al., 1999); 1:200 anti-Pax2 antibody (rabbit; Zymed); 1:300 anti-neurofilament (NF-M) antibody RMO 270.3 (mouse IgG2a; Lee et al., 1987; gift from Dr Virginia Lee); 1:500 anti-neuron-specific $\beta$ tubulin antibody TuJ1 (mouse IgG2a; BABCO); 1:100 anti-Hoxb5 antibody (rabbit; Wall et al., 1992; gift from Dr Brigid Hogan), and 1:1 anti-quail neurite QN supernatant (mouse IgG1; Tanaka et al., 1990; gift from Dr Hideaki Tanaka). Subtype-specific FITC-, TRITC-, biotinylated and horseradish peroxidase-conjugated secondary antibodies were obtained from Southern Biotechnology, Zymed and Amersham. Goat anti-rabbit Alexa ${ }^{\mathrm{TM}} 488$ (for Hoxb5) was obtained from Molecular Probes.

Embryos collected after 11 or more days of incubation were fixed overnight in Carnoy's solution (60\% ethanol, $30 \%$ chloroform, $10 \%$ acetic acid). They were dehydrated in $100 \%$ ethanol, changed into Histosol (National Diagnostics), embedded in Paraplast ${ }^{\circledR}$ (Oxford Labware) under reduced air pressure and sectioned at $10 \mu \mathrm{m}$. The sections were dried overnight at $37^{\circ} \mathrm{C}$ and stored at room temperature. Endogenous peroxidase was blocked by incubating sections for 30 minutes in $0.015 \%$ hydrogen peroxide in PBS. The sections were then processed for QCPN staining as described above, but using horseradish peroxidase-conjugated goat anti-mouse IgG1 (Zymed) at 1:200 as the secondary antibody. The colour reaction was performed in $0.05 \mathrm{mg} / \mathrm{ml}$ diaminobenzidene 3-3' tetrahydrochloride (DAB; Sigma), $0.0045 \%$ hydrogen peroxide in PBS. Gill's hematoxylin (Fisher) was used as a background nuclear stain. The slides were dehydrated and mounted in Permount (Fisher).

\section{In situ hybridisation on paraffin sections}

In situ probes for chicken Pax2 and Phox $2 a$ were obtained from Domingos Henrique and Jean-François Brunet, respectively. Wholemount in situ hybridisation was performed as described by Henrique et al. (1995). For in situ hybridisation on paraffin sections, E5.5-6.5 embryos were fixed for 4 hours in modified Carnoy's solution $(60 \%$ ethanol, $30 \%$ stock [37\%] formaldehyde [11.1\% final formaldehyde concentration], $10 \%$ acetic acid). After dehydration and paraffin sectioning at 5-6 $\mu \mathrm{m}$ as described above, a modified version of Strähle et al. (1994) and Henrique et al. (1995) was used (protocol obtained from $\operatorname{Dr} \mathrm{H}$. Etchevers, Nogent-sur-Marne, France). A detailed protocol is available on request.

For post-in situ immunohistochemistry with QCPN, slides were blocked for 30 minutes in $0.015 \%$ hydrogen peroxide in PBS. After 
three 5-minute washes in PBS (and between each subsequent antibody application), the slides were incubated in 1:1 QCPN under coverslips for 2-4 hours. After 1-2 hours under coverslip with biotinylated goat anti-mouse IgG1 (Southern Biotechnology) at 1:100, the Vectastain ABC Peroxidase standard kit (Vector Labs) was used according to the manufacturer's instructions. The DAB colour reaction was performed as described above. The slides were rinsed extensively in PBS and mounted in Fluoromount-G (Southern Biotechnology) for viewing in bright-field with Nomarski optics.

\section{RESULTS}

\section{Pax3 expression correlates with neuronal specification in opV ectoderm}

Pax3 expression in presumptive ophthalmic trigeminal (opV) placode ectoderm is first detectable by in situ hybridisation at the 4-somite stage, within the lateral ectoderm of the midbrain neural folds (Stark et al., 1997). Using the anti-neurofilament (NF-M) antibody, a very few neurofilament-positive cells are seen scattered in opV placode ectoderm as early as the 13- to 14-somite stage (J. Sechrist and M. B.-F., unpublished). Using the TuJ1 antibody, neuron-specific $\beta$-tubulin can be detected from the 19-somite stage (Moody et al., 1989). In order to test when presumptive opV placode ectoderm becomes specified to form neurons, we explanted midbrain-level ectoderm pieces from 3- to 13-somite stage quail donors and cultured them in collagen gels without serum for 20-36 hours. Although Pax3 expression is detectable by in situ hybridisation at the 4-somite stage in ectoderm above the neural tube (Stark et al., 1997), the explants were taken a little more laterally to avoid accidental inclusion of neural tissue, as in Baker et al. (1999). No Pax3 expression was seen in 3- to 4-somite stage ectoderm that was fixed immediately after explanting ( $n=10$; data not shown). Since midbrain-level neural crest cells migrate beneath presumptive opV placode ectoderm from the 8-somite stage, the donor ectoderm of 8-somite stage and older embryos was pre-labelled with DiI before explanting (Stark et al., 1997). This enabled placodal (DiI positive) neurons to be distinguished from any neural crest-derived neurons in the explants (enzyme treatment before explanting was found to be insufficient to remove all adherent neural crest cells). After sectioning, the collagen gels were immunostained with the anti-Pax 3 antibody and scored for Pax3-expressing cells, then immunostained with the antineurofilament antibody to identify neurons. The results were pooled into four time periods, viz. 3- to 4-somite stage, 5- to 7somite stage, 8- to 9-somite stage and 10- to 13-somite stage. They are presented in Fig. 1.
B showing neurofilament staining progressively increased with donor age, correlating well with the progressive increase in Pax3 expression except at the 3- to 4-somite stage. Low levels of neurofilament staining were present in a few $(<10)$ cells in $29 \%$ of 3 - to 4 -somite stage explants $(n=24)$, and a single process-bearing neuron was seen in one explant (4\%), but no Pax3 expression was detected. In our previous experiments, however, Pax 3 expression was observed in a few cells in $14 \%$ of 3- to 4-somite stage explants (Baker et al., 1999; $n=29$ ). Furthermore, a few Pax3-positive quail cells were seen in $31 \%$ of embryos after grafts of 3- to 4-somite stage quail ectoderm to the nodose region of host chick embryos $(n=13)$ (Fig. 4B; see below). This suggests that in a minority of cases, a few cells in 3- to 4-somite stage opV ectoderm are committed to Pax3 expression, correlating with the limited neurofilament staining in a few cells in a minority of the explants. However, this early commitment of a few cells to Pax 3 expression, while observed in vivo, may not have been supported in our explant conditions (similar results were obtained in studies on amphibian placode induction; Reyer, 1962). In older explants,

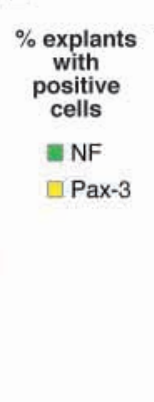

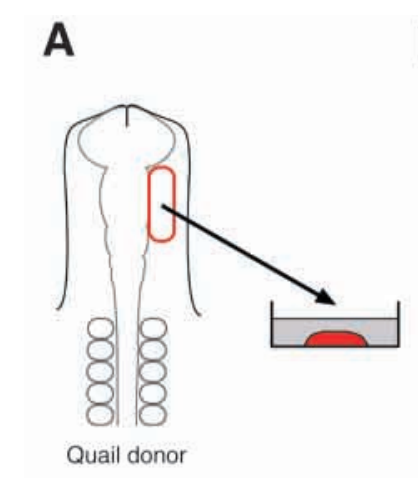
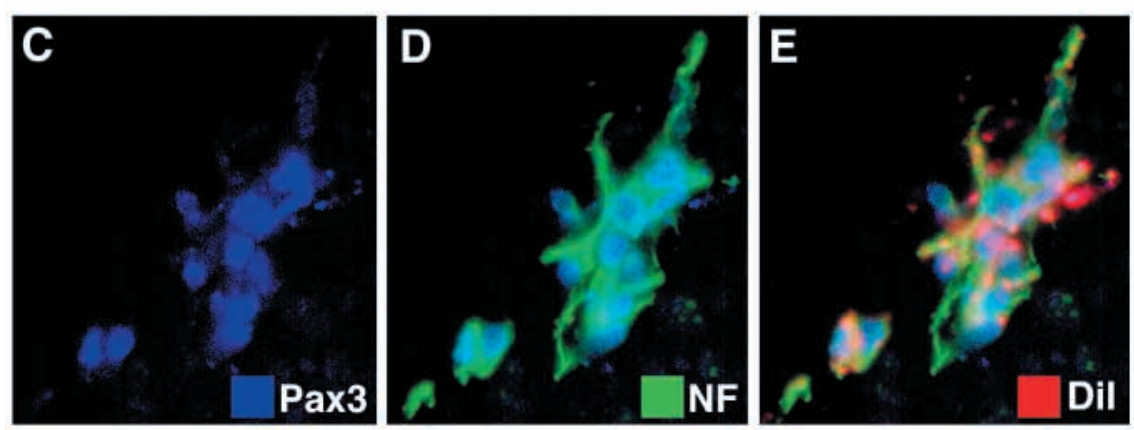

Fig. 1. Relationship between Pax3 expression and neuronal specification in explants of presumptive ophthalmic trigeminal (opV) placode ectoderm from 3-13 somite-stage quail embryos. (A) Schematic showing the midbrain-level region of ectoderm that was explanted for 20-36 hours of culture in collagen gels. After the 8-somite stage, the ectoderm was prelabelled with DiI before explanting to enable ectoderm-derived cells to be distinguished from migrating neural crest cells. The explants were sectioned and immunostained for Pax3 and neurofilament expression. (B) Histogram showing the percentage of collagen gel-cultured explants with neurofilament-positive (NF, green) and Pax3-positive cells (yellow) at different donor stages. The number of explants $(n)$ is listed at the top of each column. (C-E) Pax3-positive, DiI-positive (ectoderm-derived) neurons are seen in all 10- to 13-somite stage explants. (C) Several Pax3-positive cells (blue) seen in a 10- to 13-somite stage explant cultured for 21 hours. (D) Anti-neurofilament staining (green) shows that these Pax3-positive cells are neurons. (E) DiI-labelling (red dots) shows that these neurons are ectoderm-derived. NF, neurofilament. 
neuronal specification correlated almost exactly with Pax3 expression. In 5- to 7-somite stage explants, $45 \%$ contained a limited amount of neurofilament staining and occasional process-bearing neurons, while $40 \%$ contained a few $(<10)$ Pax3-positive cells $(n=40)$. At the 8- to 9 -stage, $72 \%$ of explants contained at least a few DiI-positive (ectoderm-derived) neurons $(n=20)$, while $81 \%$ contained at least a few Pax3-positive cells, most of which were neurons $(n=21)$. By the 10 - to 13-somite stage, all explants contained several to many $(>10)$ DiIpositive (ectoderm-derived) neurons $(n=40)$ and several to many Pax3positive cells, most of which were neurons $(n=31)$ (Fig. 1C-E). The great majority of Pax3-positive cells in the 8- to 13-somite stage explants were obviously DiI-positive and therefore ectoderm-derived, with the exception of a few Pax3-positive neurons lacking detectable DiI in two of the 10- to 13-somite stage explants $(5 \%, n=40)$. A significant increase in neuronal specification is seen at the 8-somite stage, correlating with a marked increase in Pax3-positive cells at this stage (also see Baker et al., 1999). Interestingly, opV ectoderm cells first begin to withdraw from the cell cycle at about the 8- to 10-somite stage (J. Sechrist and M. B.-F., unpublished data).

\section{Analysis of commitment of opV ectoderm by grafting it to the nodose placode}

In order to examine when presumptive opV placode ectoderm loses competence to respond to other placode-inducing signals and becomes committed to an opV fate, we challenged the ectoderm by grafting it to the nodose placode. The nodose placode, which forms the neurons of the distal ganglion of the vagal nerve, is the most caudal of the three epibranchial placodes (geniculate, petrosal and nodose). On the fate map of the 8-somite stage embryo it partially maps to the ectoderm lateral to the paraxial mesoderm, at the level of the first two somites (D'Amico-Martel and Noden, 1983). We chose the nodose placode for these experiments because it forms at some distance from the neural tube. We had previously shown that $\mathrm{Pax} 3$ is induced in unspecified opV ectoderm grafted adjacent to the neural tube at all axial levels (Baker et al., 1999). Hence, if presumptive opV placode ectoderm were grafted to any placode-forming region adjacent to the neural tube, it would be exposed to Pax3inducing signals from the neural tube in addition to the other placode-inducing signals (see Baker et al., 1999). This would make it impossible to determine whether Pax3 expression in the new location was due to prior commitment of the ectoderm, or to de novo induction of Pax3. The nodose placode forms at a sufficient distance from the neural tube to
E2.5

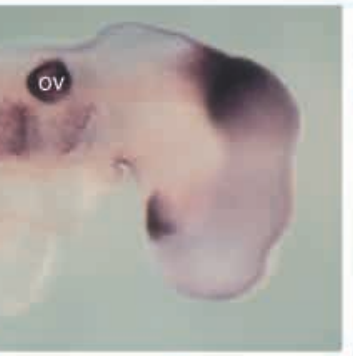
ov
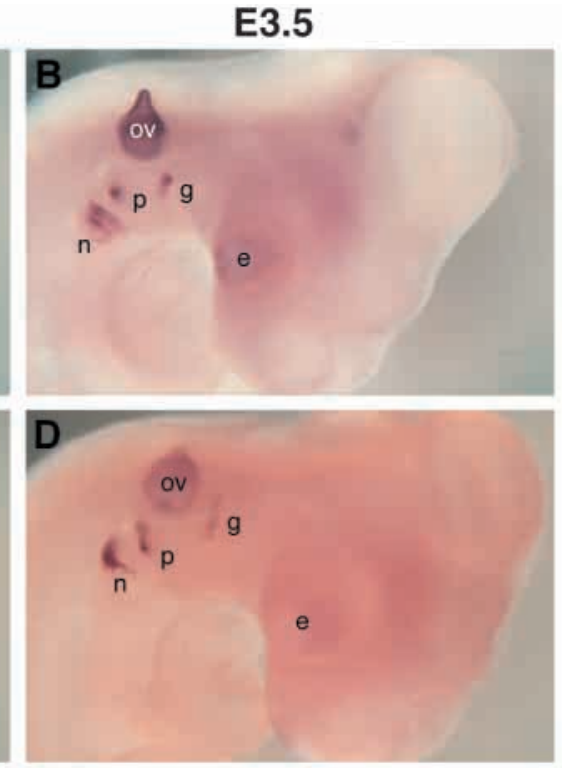

Fig. 2. Whole-mount in situ hybridisation in chick embryos for Pax2 and Phox $2 a$, two paired (hans label the epibranchial placodes and ganglia. (A) Pax2

D) Phox $2 a$ expression at E3.5 in the three epibranchial placode-derived ganglia. Faint staining in the otic vesicle is substrate trapping.

avoid de novo induction of Pax3 in opV ectoderm grafted to that location (also see Baker et al., 1999).

We used the paired homeodomain transcription factor Pax2 as an early marker for nodose placode ectoderm, and the paired homeodomain transcription factor Phox $2 a$ as a marker for nodose placode neurons (Begbie et al., 1999; Valarché et al., 1993). As previously described, Pax2 is expressed at early stages in the otic placode in the chick (Herbrand et al., 1998). However, it is also expressed at E2.5 in a broad band of ectoderm encompassing the ectoderm fated to form the three epibranchial placodes (Fig. 2A; D'Amico-Martel and Noden, 1983). By E3.5, it is expressed specifically in the otic vesicle and the ectoderm of the forming epibranchial placodes, including the nodose placode (Fig. 2B). This can be compared to the expression of Phox2a, which in the head specifically marks neurons of the three epibranchial placodes (Valarché et al., 1993; Morin et al., 1997; Fig. 2C,D).

As a control for Pax 2 expression in quail ectoderm grafted to the nodose region, we isolated presumptive nodose placode ectoderm (lateral to the first two somites) from dispase-treated 8-9 somite stage quail embryos. We grafted it isotopically to the same region of host chick embryos (Fig. 3A): the grafted quail ectoderm expressed high levels of Pax2 at E2.5 ( $n=2$; Fig. 3B,C).

In order to address the competence of opV ectoderm to express Pax2 in response to epibranchial placode-inducing signals, 3- to 16-somite stage opV ectoderm was grafted lateral to the first two somites at the 3- to 10-somite stage and the embryos fixed 16-31 hours later (Fig. 4A). After sectioning, we looked for Pax2-positive quail ectoderm cells and/or for 
A

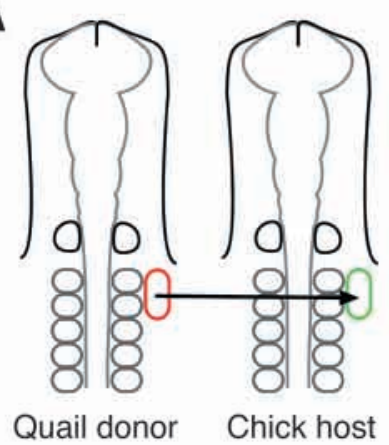

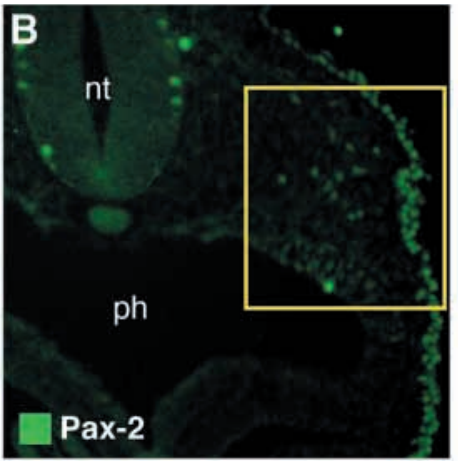

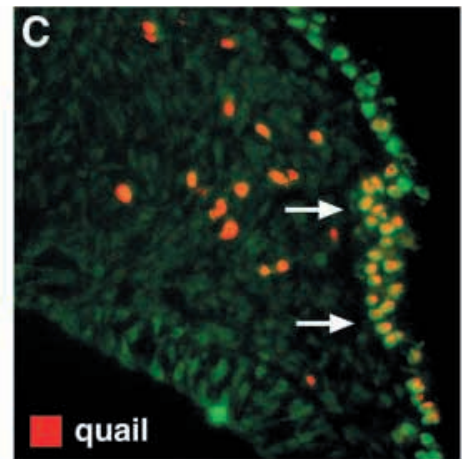

Fig. 3. Presumptive nodose placode ectoderm grafted isotopically from quail to chick embryos expresses Pax 2 at E2.5. (A) Schematic showing the region of ectoderm grafted. (B) Transverse section through the nodose placode region of a chick host collected 23 hours after an isotopic, isochronic graft of 8-somite stage quail nodose placode ectoderm. Pax 2 expression is green. (C) Higher-power view of the boxed area in $\mathrm{B}$, showing QCPN-positive quail cells in red. Pax2-positive quail cells in the ectoderm are yellow (arrows). nt, neural tube; ph, pharynx.

Pax3-positive quail cells in the ectoderm and mesenchyme. The results are presented graphically in Fig. 4B, and examples shown in Fig. 4C-G. In all grafts, a substantial region of host placode remained, suggesting that the nodose placode was not completely replaced by the grafted opV ectoderm. Only those grafts that were incorporated

Fig. 4. Presumptive opV placode ectoderm from quail donors progressively loses competence to express Pax2 when grafted to the nodose placode of chick hosts.

(A) Schematic diagram showing the graft procedure.

(B) Histogram showing the percentage of chimeras with Pax2-positive and Pax3-positive quail cells 16-31 hours after grafts from different donor stages. Donor stage, somite stage. The number of grafts (n) is listed at the top of each column. Pax 2 expression (green) declines as Pax3 expression (yellow) increases within the grafted ectoderm. By the 14- to 18-somite stage, fewer than five Pax2-positive cells were seen per embryo.

(C) Transverse section through the nodose placode region of a chick host collected 20.5 hours after a graft of 4-somite stage quail midbrain-level ectoderm. Pax2 expression is green. (D) Higher-power view of the boxed area in C, showing QCPN-positive quail cells in red and Pax 2 in green. Pax2-positive quail cells in the ectoderm are yellow (arrows). Yellow cells in the aorta (a) are blood cells. (E-G) Older opV ectoderm is less competent to express Pax 2 and expresses more Pax3. These panels show a transverse section through the nodose placode region of a chick host collected 21 hours after receiving a graft of 12-somite stage opV placode ectoderm. (E) Pax3-positive cells (green) in the ectoderm and in the mesenchyme (arrowheads). Arrow indicates Pax3-positive cells that are also Pax2-positive (see also F and G). (F) Pax2 expression (blue) in the ectoderm. (G) QCPN-positive quail cells in red (the ectoderm was prelabelled with DiI before grafting, also seen in red), together with Pax3 (green) and Pax2 (blue). The Pax3-positive cells in the mesenchyme and ectoderm are seen to be quail cells (arrowheads). Arrow indicates two Pax $2^{+} \mathrm{Pax} 3^{+}$quail cells in the ectoderm. Note that the majority of the grafted ectoderm is Pax2negative, sandwiched between Pax $2^{+}$host ectoderm. This was the only case seen of $\mathrm{Pax} 2^{+} \mathrm{Pax} 3^{+}$cells. a, aorta; nt, neural tube; dm, dermomyotome.
A
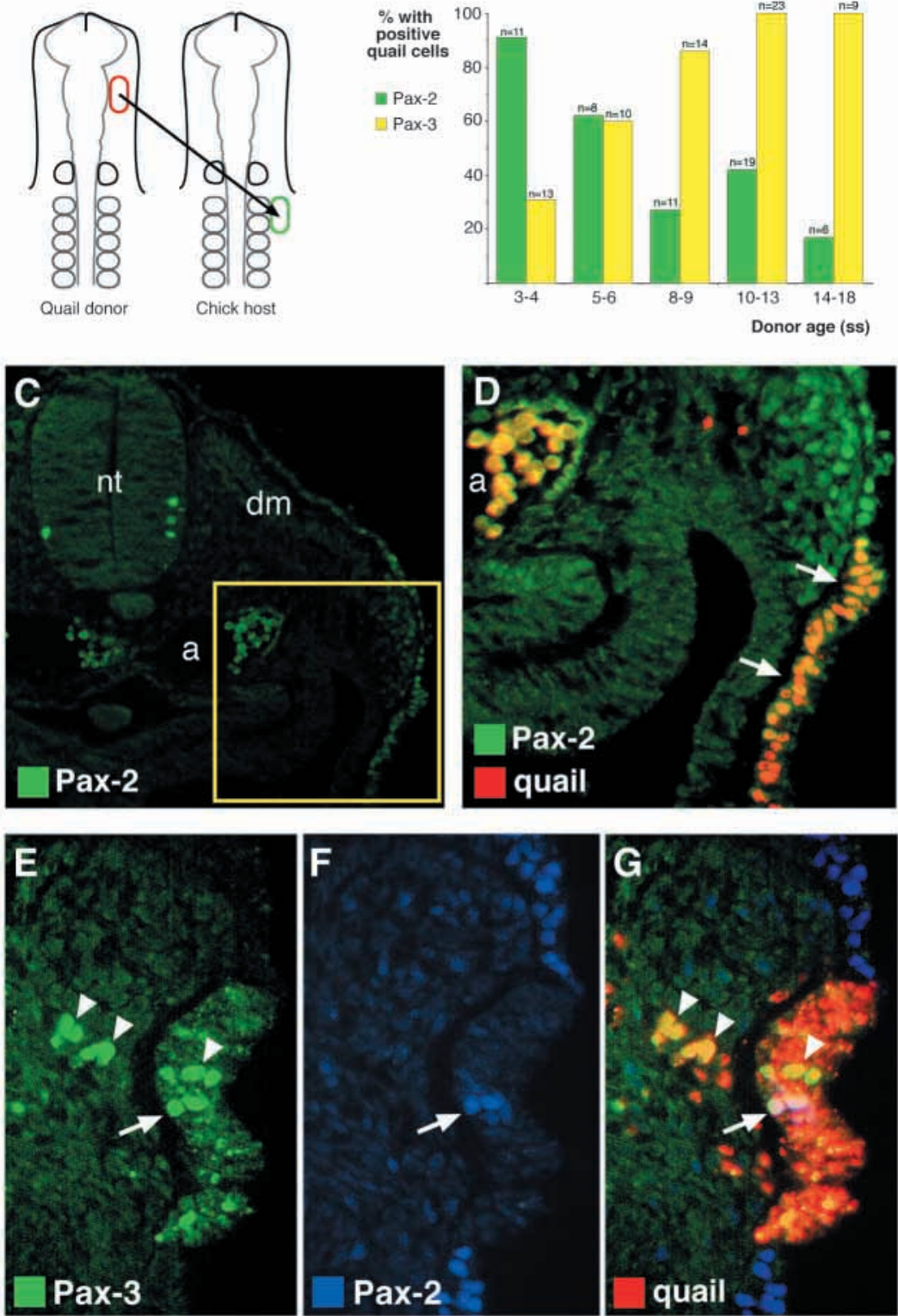
within the host Pax2-positive region were scored for Pax2 expression. The results show that competence to respond to Pax2-inducing signals declined as Pax3 expression increased, with increasing donor age (Fig. 4B). The Pax3 expression in these embryos reflects Pax3 induction in the ectoderm prior to grafting, and parallels the Pax 3 commitment data obtained previously by grafting the ectoderm over the lateral plate mesoderm in the trunk (Baker et al., 1999). 91\% of grafts of 3- to 4-somite stage opV ectoderm contained at least a few $(<10)$ and usually many Pax2-positive quail ectoderm cells at E2.5 ( $n=11)$ (Fig. 4C,D), while only $31 \%$ contained any $(<5)$ Pax3-positive cells $(n=13)$. The Pax2-positive cells were generally grouped together, as seen in Fig. 4D, rather than being scattered in the ectoderm. Presumptive opV ectoderm taken at the 5- to 6-somite stage gave rise to at least a few Pax2positive quail ectoderm cells in $62 \%$ of cases $(n=8)$, and to at least a few Pax3-positive quail cells in $60 \%$ of cases $(n=10)$. After grafts of 8-somite stage and older opV ectoderm, most embryos showed no Pax2 expression at all. At the 8- to 9somite stage, only $27 \%$ of grafts formed any Pax2-positive quail ectoderm cells $(n=11)$, while $86 \%$ contained at least a few Pax3-positive quail cells $(n=14)$. After grafts of 10-somite stage and older opV ectoderm, there were fewer than five Pax2positive cells in $42 \%$ of 10 - to 13 -somite stage ectoderm grafts ( $n=19)$ (Fig. 4F,G), and $17 \%$ of 14 - to 18 -somite stage ectoderm grafts $(n=6)$. In contrast, all embryos that received grafts of 10-somite stage and older opV ectoderm contained at least a few Pax3-positive cells (total $n=32$ ) (Fig. 4E-G). Interestingly, out of 17 embryos that contained both Pax3positive and Pax2-positive quail cells at E2.5, only two $\mathrm{Pax} 2^{+} \mathrm{Pax} 3^{+}$quail cells were seen. These were both in one section of an embryo that had received a graft of 12-somite stage opV ectoderm (Fig. 4E-G). Together with the robust expression of Pax 3 in older opV ectoderm, this suggests that co-expression of Pax 3 and Pax 2 is a rare and/or transient event.

After the 8-somite stage, the grafts contained mesencephalic neural crest cells, which migrate beneath the opV ectoderm. It was difficult to remove these neural crest cells even with dispase treatment. However, the presence of mesencephalic neural crest cells did not seem to affect the expression of Pax2 in the ectoderm, as competence to express Pax 2 began to fall before neural crest cells were present in the grafts (i.e. before the 8-somite stage), and extended for some time afterwards. Mesencephalic neural crest cells down-regulate Pax3 expression after the initial stages of their migration, such that high levels of Pax3 are only seen in placode-derived cells at E2.5 (see Baker et al., 1999; Stark et al., 1997). Mesenchymal Pax3-positive quail cells were seen both scattered as individual cells or in small clusters (e.g. Fig. 4E-G).

As a further control for the presence of neural crest cells in the grafts after the 8-somite stage, we performed 'double grafts'. In these experiments, 4- to 6-somite stage opV quail ectoderm was grafted isotopically and isochronically into chick hosts (i.e. before neural crest cell migration). The first hosts were incubated to the 10- to 13-somite stage, and then the same region of ectoderm was re-grafted from these embryos to the nodose region of a second host. Any neural crest cells present in the graft are of chick origin, from the first host; thus, the only quail cells present are ectoderm-derived. Pax3-positive quail cells were seen at E2.5 in the mesenchyme of four out of five such double-grafted embryos. They were found in similar
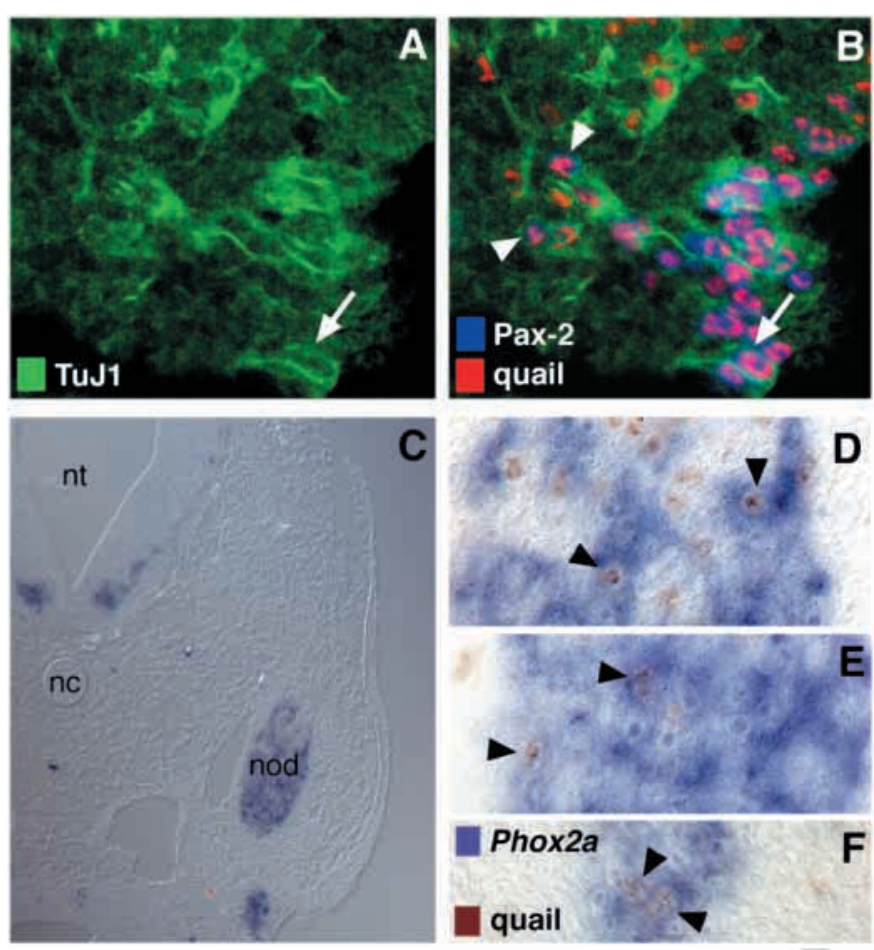

Fig. 5. Young opV ectoderm grafted to the nodose placode expresses Pax 2 and forms Phox $2 a$-positive neurons in the nodose ganglion by E3.5. (A) Transverse section through the nodose placode region of a chick host collected 2 days after receiving a graft of 5-somite stage opV placode ectoderm. TuJ1-positive neurons are green. (B) Same section as in A, showing QCPN-positive quail cells in red, Pax2positive cells in blue (all the Pax2-positive cells are quail cells and therefore pink), and TuJ1-positive neurons in green. At least one Pax2-positive quail neuron can be seen in the ectoderm (arrow), together with Pax2-positive quail cells in the mesenchyme (arrowheads). (C) Transverse section through the nodose placode region of a chick host collected 2 days after receiving a graft of 6somite stage opV placode ectoderm. Phox $2 a$ expression (purple) was detected by in situ hybridisation on the section, while quail cells were subsequently detected by QCPN immunohistochemistry (brown precipitate). nc, notochord; nod, nodose ganglion; nt, neural tube.

(D-F) Higher power views of Phox $2 a$-positive quail cells

(arrowheads). D and $\mathrm{E}$ are from the same embryo as shown in $\mathrm{C} ; \mathrm{F}$ is from an embryo that received a graft of 4-somite stage opV placode ectoderm.

numbers and arrangements as were seen in the single grafts of opV ectoderm after the 8-somite stage (data not shown).

These results suggest that as the number of Pax3-positive cells increases in opV ectoderm, it becomes increasingly committed to Pax3 expression and concomitantly loses competence to respond to Pax2-inducing signals present at the level of the nodose placode.

\section{OpV ectoderm forms neurons in the nodose ganglion}

We used the TuJ1 anti-neuron-specific $\beta$-tubulin antibody to look for quail neurons in sections of E3.5 chimeras that had received 3- to 4-somite stage opV ectoderm grafts to the nodose region at the 3- to 8-somite stage. At E3.5, 96\% of 3to 4-somite stage opV quail ectoderm grafts had formed neurons in the forming nodose and/or petrosal ganglia $(n=24)$. 
The final position of some of the grafts evidently extended sufficiently rostrally for the ectoderm to contribute neurons not only to the nodose but also to the petrosal ganglion (for fatemap, see D'Amico-Martel and Noden, 1983). As shown above, most 3- to 4-somite stage opV ectoderm grafts contained at least some Pax2-positive cells at E2.5 $(91 \% ; n=11)$, while most $(69 \%)$ contained no Pax3-positive cells $(n=13)$. Similarly, all 4-somite stage opV ectoderm grafts analysed for Pax2 at E3.5 contained substantial amounts of Pax2-expressing ectoderm $(n=8)$, while the majority $(75 \%)$ contained no Pax-3-positive cells $(n=8)$. Very few, if any, neurons are specified in 3- to 4somite stage opV ectoderm (see above); no Pax3 is induced de novo in the nodose placode region, and the majority of 3- to 4somite stage opV ectoderm grafts formed no Pax3-positive cells at E2.5 or E3.5. However, almost all 3- to 4-somite stage opV ectoderm grafts formed neurons in the nodose and/or petrosal ganglia at E3.5. These results suggest that the majority of these quail neurons were derived from ectoderm that had been induced to express Pax 2 . Indeed, in one embryo, Pax2 was seen faintly in both host and graft-derived cells that had delaminated from the nodose placode, including at least a few neurons in or near the ectoderm (Fig. 5A,B). Hence, 3- to 4somite stage opV ectoderm grafted to the nodose region both expresses the epibranchial placode marker Pax 2 and forms neurons in the nodose ganglion that are likely to be derived from Pax2-expressing cells.

\section{OpV ectoderm-derived neurons in the nodose ganglion express Phox2a}

To determine whether the grafted ectoderm forms neurons of appropriate character in the nodose ganglion, we used the paired homeodomain transcription factor gene Phox $2 a$ as a marker (Valarché et al., 1993). This gene is expressed by and is essential for the development of epibranchial placodederived neurons (Fig. 2C,D) (Morin et al., 1997; Valarché et al., 1993), but it is not expressed in any part of the trigeminal ganglion. When 4- to 6-somite stage opV ectoderm was grafted to the nodose region of 6- to 8-somite stage host embryos, $88 \%$ contained at least a few clearly Phox $2 a$ positive quail cells within the nodose ganglion at E3.5 ( $n=8$; Fig. 5C-F). Not all quail cells within or associated with the ganglion were Phox $2 a$-positive. Our results therefore confirm that 4- to 6-somite stage $\mathrm{op} \mathrm{V}$ ectoderm is competent to give rise to neurons of epibranchial character in the nodose ganglion.

\section{OpV ectoderm is not competent to express the nodose-specific marker Hoxb5}

The results described above show that when grafted to the nodose region, 4-somite stage opV ectoderm does not express the opV marker Pax3. It is competent to express the epibranchial placode marker Pax2, and forms Phox $2 a$-positive neurons in the nodose ganglion. However, Pax 2 and Phox $2 a$ are general epibranchial placode markers, rather than nodose placode-specific markers. Hoxb5 is expressed at E3.5 in nodose placode and neurons, but not in any of the other, more rostral placodes (Kuratani and Wall, 1992; Wall et al., 1992). Although Hoxb5 does not seem to be necessary for the formation of nodose neurons (Rancourt et al., 1995), we used it as a molecular marker for nodose placodederived neurons at E3.5-E4.5.

In control experiments, four out of five isotopic grafts of 4to 8-somite stage presumptive nodose placode ectoderm (lateral to the first two somites) formed many Hoxb5-positive neurons in the nodose ganglion at E3.5 (Fig. 6A-C). Similarly,
A

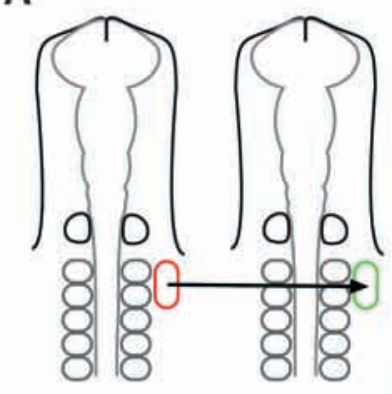

Quail donor

D

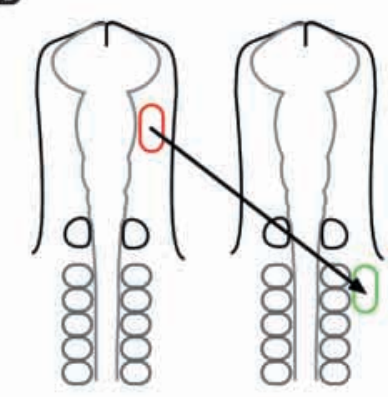

Quail donor
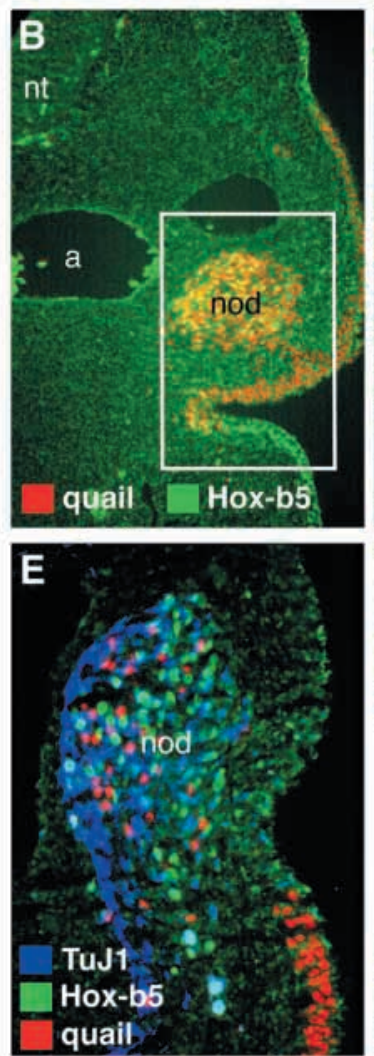
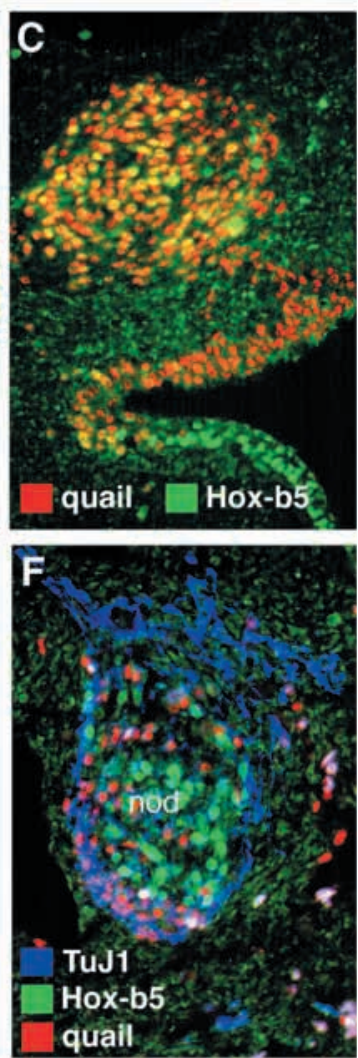

Fig. 6. Presumptive nodose placode ectoderm (A-C), but not presumptive opV placode ectoderm (D-F), is competent to express the nodose-specific marker Hoxb5.

(A) Schematic showing control isotopic grafting procedure for presumptive nodose placode ectoderm. (B) Transverse section through the nodose placode region of a chick host collected 2 days after receiving a graft of 8 -somite stage nodose placode ectoderm. QCPN-positive quail cells are red; Hoxb5-positive cells are green. (C) Higher-power view of the boxed area in B. QCPN-positive quail cells are red; Hoxb5-positive cells are green. Hoxb5-positive quail cells are yellow. (D) Schematic diagram showing grafting procedure for opV ectoderm grafted to the nodose placode. (E) Transverse section through the nodose placode region of a chick host collected 2 days after receiving a graft of 5-somite stage opV placode ectoderm. QCPN-positive quail cells are red; Hoxb5-positive cells are green; TuJ1-positive neurons are blue. No Hoxb5-positive quail cells are seen. nod, nodose ganglion. (F) Transverse section through the nodose placode region of a chick host collected 2 days after receiving a graft of 4-somite stage opV placode ectoderm. QCPNpositive quail cells are red; Hoxb5-positive cells are green; TuJ1-positive neurons are blue. No Hoxb5-positive quail cells are seen. Whitish cells are blood cells. nod, nodose ganglion. a, aorta; nod, nodose ganglion; nt, neural tube. 
all grafts of 4- to 6-somite stage oticlevel ectoderm (which normally does not express Hoxb5) gave rise to at least a few Hoxb5-positive cells in the nodose ganglion ( $n=7$; data not shown). This shows that non-nodose ectoderm can be induced to express Hoxb5 when grafted to the nodose placode region. When 3- to 4-somite stage opV ectoderm was grafted to the nodose region, however, Hoxb5positive quail cells were never seen at E3.5, although quail neurons were always present $(n=10)$. Three of the donor embryos for these grafts were dispase-treated to ensure removal of any midbrain-level cephalic mesoderm that might affect Hox gene induction in the grafted ectoderm (Gould et al., 1998; Itasaki et al., 1996; Trainor and Krumlauf, 2000). Similarly, 5- to 6somite stage opV ectoderm grafted to the nodose region did not express Hoxb5 at E3.5-E4.5, but formed neurons in the nodose ganglion in $88 \%$ of cases ( $n=8$; Fig. 6D-F). All of these donor embryos were dispase-treated to ensure removal of any contaminating midbrain-level cephalic mesoderm.

Taken together, our results suggest that opV ectoderm taken even before it expresses any significant amount of Pax3 (at the 3- to 4-somite stage), is competent to express the early epibranchial placode marker Pax 2 and to form Phox $2 a$-positive neurons in the nodose ganglion. However, neither the grafted ectoderm nor graft-derived neurons in the nodose ganglion express Hoxb5, which is expressed in nodose neurons (Kuratani and Wall, 1992; Wall et al., 1992) but does not seem to be required for their formation (Rancourt et al., 1995). This suggests that even at the 3- to 4-somite stage, presumptive opV ectoderm is qualitatively different from more caudal ectoderm such that it does not respond to Hoxb5-inducing signals present in the nodose placode region.

\section{OpV ectoderm-derived neurons show nodose-like central and peripheral projections and survive in the nodose ganglion until at least E12.5}

Given the lack of Hoxb5 expression in opV ectoderm grafted to the nodose region, it was important to characterise further the nature of the neurons derived from this ectoderm. We
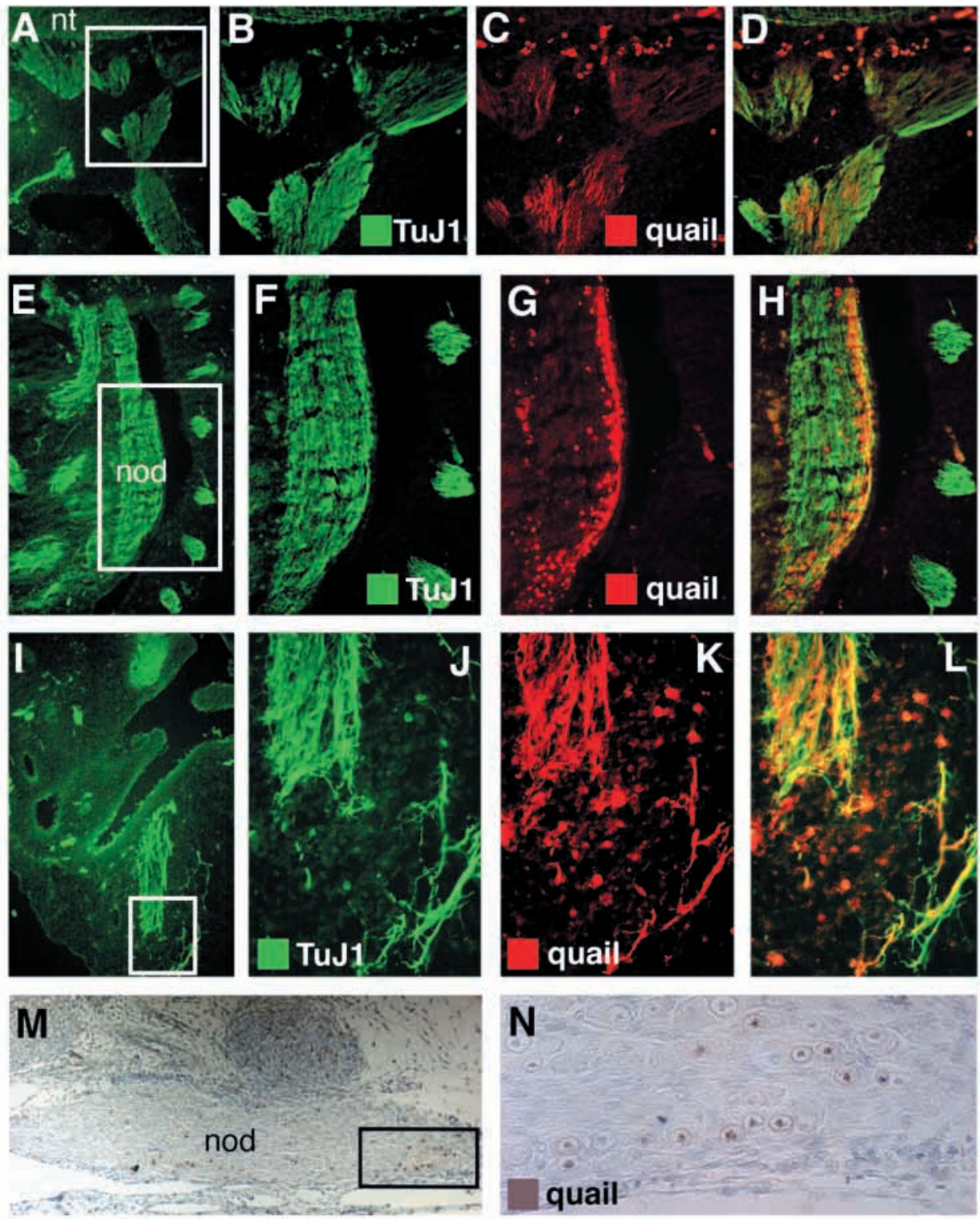

Fig. 7. OpV ectoderm-derived neurons in the nodose ganglion project centrally and peripherally together with surrounding host neurons at E5.5 (A-L). They survive in the nodose ganglion until at least E12.5, well after the main period of cell death in the ganglion (M,N). (A) Parasagittal section through the nodose ganglion root region of a host chick embryo at E5.5, 4 days after receiving a graft of 5-somite stage opV ectoderm. TuJ1-positive neurons are green. (B) Higherpower view of the boxed area in A. (C) Same view as B, but showing QN-positive quail neurites and QCPN-positive quail cells in red. (D) Merging the colour channels shows quail neurites projecting centrally to the hindbrain together with surrounding host neurites. (E) Parasagittal section through the nodose ganglion (nod) of a different host chick embryo, 4 days after receiving a graft of 5-somite stage opV ectoderm. TuJ1-positive neurons are green. (F) Higherpower view of the boxed area in E. (G) Same view as F, but showing QN-positive quail neurites and QCPN-positive quail cells in red. (H) Merging the colour channels shows quail neurites following surrounding host neurites. (I) Parasagittal section through the peripheral projections of the nodose ganglion of the same embryo as in A-D. TuJ1-positive neurons are green. (J) Higher-power view of the boxed area in I. (K) Same view as J, but showing QN-positive quail neurites and QCPN-positive quail cells in red. (L) Merging the colour channels shows quail neurites projecting together with host neurites. (M) Parasagittal section through the nodose ganglion (nod) of a chick host embryo at E12.5, 11 days after receiving a graft of 4somite stage opV ectoderm. QCPN-positive quail cells are brown. (N) Higher-power view of the boxed area in M, showing several quail cells within the ganglion. nod, nodose ganglion; nt, neural tube. 
therefore examined the central and peripheral projections of nodose ganglion neurons derived from grafts of 5-somite stage opV ectoderm, by immunostaining parasagittal sections of E5.5 embryos $(n=5)$ with the anti-quail neurite antibody QN (Tanaka et al., 1990). We also analysed the long-term survival of these neurons in the nodose environment.

As shown in Fig. 7A-L, 5-somite stage quail opV ectoderm grafted to the nodose region of 10-somite stage chick hosts, had formed many neurons in the nodose ganglion at E5.5. Their neurites associated with host nodose neurites along normal pathways centrally to the hindbrain (Fig. 7A-D), within the main body of the ganglion (Fig. 7E-H), and peripherally (Fig. 7I-L). Furthermore, 4-somite stage quail opV ectodermderived neurons survived in the nodose ganglion until at least E12.5. This is after nodose cell numbers have stabilised at E11, and well after the peak of cell death in the ganglion at E7 (Hiscock and Straznicky, 1986) (n=2; Fig. 7M,N). This suggests that the growth factor requirements of the opV ectoderm-derived neurons were met in the nodose environment. Taken together with the expression of the epibranchial placode marker Pax 2 and the epibranchial neuron marker Phox $2 a$, these results suggest that the grafted opV ectoderm has formed epibranchial neurons that are most likely nodose in character.

\section{Pax3-expressing opV ectoderm follows a primarily opV fate in the nodose region}

As described above, older opV ectoderm that already contains significant numbers of Pax3-positive cells (10-18 somite stage), is much less competent to express Pax2 than is the mostly Pax3-negative 3- to 4-somite stage opV ectoderm. 60\% of 10- to 18-somite stage opV ectoderm grafts showed no Pax2 expression at E2.5, and fewer than five Pax2-positive cells were seen per embryo in the remaining $40 \%(n=25)$. In order to follow the longer-term fate of 10 - to 18-somite stage opV ectoderm in the nodose region, it was necessary to distinguish placode-derived cells from the mesencephalic neural crest cells that are also present in the grafts (see above). Dispase treatment before explant was not always sufficient to remove all adherent neural crest cells. Hence, either the surface ectoderm of donor embryos was labelled with DiI prior to ectoderm removal (Stark et al., 1997), or a 'double graft' was performed (see above).

At E2.5, 80\% of grafts of 10- to 14somite stage double-grafted quail 4- to 6-somite stage opV ectoderm had formed neurons $(n=5)$. This is consistent with an opV fate, as opV neurons differentiate from $\mathrm{E} 2$, while nodose neurons are not usually seen in significant numbers until E3.5. All grafts of 9- to 17-somite stage ectoderm that were analysed for Pax 3 expression had formed clearly Pax3-positive neurons by $\mathrm{E} 2.5(n=7)$, again consistent with an opV fate (Fig. 8A-C). A few Pax3-negative neurons were also seen in three of these grafts, including one graft of 17-somite stage ectoderm.

At E3.5, all grafts of DiI-labelled 13 - to 16-somite stage quail opV ectoderm had formed DiI-positive quail neurons in the nodose and/or petrosal ganglia ( $n=6$; data not shown). Pax3 expression was only seen in a few quail cells in $29 \%$ of 11 - to 16 somite stage grafted embryos at E3.5 $(n=7)$, suggesting that Pax3 was being down-regulated by E3.5. Although Pax3 expression levels are still high in the opV ganglion at E3.5, isotopic grafts of quail opV ectoderm show that some quail cells in the opV ganglion do not express Pax3 at E3.5 (data not shown). This suggests that Pax 3 is also being down-regulated in the opV ganglion by E3.5. 
To determine whether the grafted opV ectoderm formed any epibranchial-character neurons in the nodose ganglion, we used Phox $2 a$ as a marker (see above). A few Phox $2 a$-positive quail cells were seen in $57 \%$ of 14- to 16-somite stage grafted embryos at E3.5 $(n=7)$. However, where present, there were fewer than five such cells per embryo and they were much harder to find than in the 4- to 6-somite stage grafted embryos (Fig. 5D-F) (data not shown). Hence the great majority of neurons derived from older opV ectoderm at E3.5 are Phox $2 a$ negative and thus not of epibranchial character. It was interesting to note that these neurons were generally segregated from host nodose neurons at E3.5 (Fig. 8D-F), while quail neurons derived from grafts of younger opV ectoderm were well integrated in the host nodose ganglion (Fig. 6D-F). As was the case for 3- to 6-somite stage opV ectoderm, Hoxb5 expression was not seen at E3.5 after grafts of older (15- to 16somite stage) opV ectoderm ( $n=3)$ (Fig. $8 \mathrm{D}-\mathrm{F})$.

It is likely that the few Phox $2 a$-positive cells that were sometimes present in the nodose ganglion at E3.5 are derived from the few Pax2-expressing cells sometimes seen at E2.5 (see above). A few neurons from a 14-somite stage opV ectoderm graft survived until at least E11.5, after the main period of cell death in the ganglion (data not shown). Again, these neurons were probably derived from the few cells that adopted a nodose fate. The many Pax3-expressing neurons seen at E2.5 (Fig. 8A-C) are presumably unable to survive in the nodose environment, although we did not investigate this further.

\section{DISCUSSION}

\section{Stages in placodal commitment}

We have shown that very few, if any, cells in 3- to 4-somite stage opV ectoderm are specified to form neurons, or to express the opV marker Pax3. At the 3- to 4-somite stage, opV ectoderm is competent to express epibranchial placode and neuron markers (Pax2 and Phox $2 a$ ) when grafted to the nodose region. This competence declines with time as the number of Pax3-expressing cells increases in the ectoderm. Competence is essentially lost by all but a few cells by the 14- to 18 -somite stage, when many cells in the ectoderm are already expressing Pax3, and when neuronal markers are first seen in the ectoderm in vivo. Vogel and Davies (1993) similarly found that presumptive chick otic and nodose placodes at the 4- to 7somite stage, which are not yet specified to form neurons, could be interchanged. In both cases, the grafted ectoderm formed neurons appropriate to its new location, in terms of their hindbrain innervation pattern, neurite growth and cell survival characteristics. Given that these placodes both express Pax2, it would be interesting to discover whether the period of competence for substitution is extended in these placodes as compared with the opV placode.

Székely (1959) exchanged the trigeminal and vagal (nodose) placodes between late neurula stage Triturus and Pleurodeles embryos. Unusually, Székely used a behavioural response as his assay for neuronal fate. In the larval animal, the vagal nerve mediates the gill depression reflex. When the trigeminal placode was substituted for the vagal placode, there was a normal gill reflex after gill stimulation. Hence, the heterotopic trigeminal placode established connections appropriate to its new location, in agreement with the results obtained here using young (3- to 4-somite stage) opV ectoderm.

In late neurula stage amphibian embryos, Jacobson (1963c) reversed the anteroposterior polarity of presumptive placodal ectoderm and found that presumptive otic placode ectoderm located rostrally formed both ear and nose. Similarly, presumptive olfactory placode located caudally formed both nose and ear. He concluded that the ectoderm was sufficiently committed to its original fate to express that fate ectopically, but that it was still labile enough to respond to new inducing activity. This seems to correspond to our results with older opV ectoderm. Some cells in the older opV ectoderm were already committed to express Pax3 (the opV placode marker), while other cells were not yet committed and could be induced to express Pax2 (the epibranchial placode marker). Similarly, some cells in the opV ectoderm are still cycling while others are post-mitotic (J. Sechrist and M.B.-F., unpublished data). Hence, the 'committed but labile' nature of the ectoderm in Jacobson's experiments is most easily explained by the existence of two distinct cell populations within the ectoderm. Some cells were already committed to their original fate (e.g. otic), while those that were not yet so committed could be induced to adopt a different fate (e.g. nose).

The induction of different fates in the same piece of ectoderm can also be explained by the exposure of different regions of ectoderm to different signals. This is unlikely to explain Jacobson's experiments since this would imply that noses and ears should form both rostrally and caudally in the normal embryo. However, we have seen this result in previous experiments, where we took presumptive opV placode ectoderm before it contained significant numbers of Pax3positive cells and grafted it to the otic placode region (Baker et al., 1999). The region of grafted ectoderm that lay adjacent to the neural tube expressed Pax3 in response to the neural tube-derived signal (Baker et al., 1999; Stark et al., 1997). However, more lateral ectoderm did not express Pax3 and instead contributed to the otic placode, presumably because it had greater exposure to otic placode-inducing signals (Baker et al., 1999).

\section{Neurogenesis and neuronal subtype identity are linked}

Stone (1924) found that the opV placode in early tailbud stage Ambystoma embryos (stage 26, i.e. 10- to 12-somite stage; Armstrong and Malacinski, 1989) was already committed to a neuronal fate, as it formed a ganglion when transplanted to the flank. It was able to substitute for the gasserian (maxillomandibular trigeminal; $\mathrm{mmV}$ ) placode, forming a ganglion that seemed to replace entirely the gasserian in form and function (Stone, 1924). However, when it was substituted for various lateral line placodes, it formed a small ganglion whose nerve fibres often followed lateral line and other cranial nerves, but never actually innervated lateral line organs (Stone, 1928). Similarly, pre- and postotic lateral line placodes, taken after commitment to neurogenesis, were able to substitute for each other but not for the opV placode (Stone, 1929).

Stone's results show that after commitment to neurogenesis, placodes of similar function (opV and $\mathrm{mmV}$; pre- and postotic lateral line placodes) could substitute for one another, but not for placodes that perform different functions. Our results, and those of Vogel and Davies (1993), show that before 
commitment to neurogenesis, presumptive placodal ectoderm cells are competent to contribute appropriately to placodes of different function. Taken together, these data suggest that neuronal differentiation in the neurogenic placodes is tightly linked to neuronal subtype.

There is increasing evidence that in both vertebrates and invertebrates, homologous basic helix-loop-helix (bHLH) proneural transcription factors specify both neurogenesis and context-dependent neuronal subtype identity (reviewed by Anderson and Jan, 1997; Brunet and Ghysen, 1999). Proneural gene specification of neuronal subtype identity commonly occurs via the activation of paired homeodomain transcription factors that directly regulate neurotransmitter enzyme gene expression (Dambly-Chaudière et al., 1992; Miller et al., 1992; Vervoort et al., 1995; Zhao and Emmons, 1995; reviewed by Anderson and Jan, 1997). In Xenopus, the bHLH genes Xath3 and $X$-ngnr- 1 have generic neuronal-inducing activity and also induce the context-dependent development of sensory-specific neuronal subtypes (Perron et al., 1999). In the chick, the bHLH neurogenin genes instructively induce both panneuronal and sensory-specific neuronal markers in the neural crest, as well as ectopically in the dermomyotome (Perez et al., 1999). BMP2-mediated induction of the bHLH gene Mash1 in neural crest cells leads both to generic neuronal differentiation and acquisition of sympathetic neurotransmitter identity, via experimentally separable subprograms that are linked by the Phox2a gene (Groves et al., 1995; Sommer et al., 1995; Shah et al., 1996; Lo et al., 1998, 1999). In the epibranchial placodes, the bHLH gene neurogenin 2 (ngn2) is essential for neuronal differentiation, but not for Phox $2 a$ expression, in the geniculate and petrosal placodes (Fode et al., 1998). Phox2a expression is required for transient dopamine $\beta$-hydroxylase expression (Morin et al., 1997). Interestingly, the epibranchial placode cells that express Phox2a also express the highest levels of $n g n 2$ transcripts, suggesting that the same signal might be involved in the induction of both genes, coupling the generic and neuronal identity subprograms (Lo et al., 1999). Indeed, in explant experiments in the chick, BMP7 appears to be able to induce Phox2a-positive neurons directly from nonepibranchial placode head ectoderm (Begbie et al., 1999), while the BMP7 inhibitor follistatin reduces the induction of Phox 2a-positive neurons in head ectoderm by pharyngeal endoderm (Begbie et al., 1999).

\section{Function of Pax3 in the opV placode}

The splotch mouse, which has a mutation in the Pax3 gene, has a reduction in the opV nerve (Tremblay et al., 1995). Cranial neural crest migration and contribution to the trigeminal ganglion appear to be normal in these mice, however (Serbedzija and McMahon, 1997). Since Pax3 is also expressed in the mouse trigeminal placode region (Stark et al., 1997), the opV defect in the mutant mice is presumably due to the loss of placodal Pax3 expression. This suggests that Pax3 function is indeed necessary for the formation of the placodal component of the opV ganglion. As our results suggest a correlation between Pax 3 expression and neurogenesis in the opV placode, it is tempting to speculate that Pax3 may also specify neuronal phenotype, like Phox2a in the epibranchial placodes. In neural crest cells, Pax3 is an important regulator of sensory neurogenesis (Koblar et al., 1999). Sensory neurogenesis is reduced in neural crest cultures from splotch mice, and antisense oligonucleotide treatment of dorsal root ganglion cultures against Pax3 also decreases sensory neurogenesis (Koblar et al., 1999).

\section{Early regionalisation of competence in head ectoderm}

Our results further show that different regions of head ectoderm at the 3- to 4-somite stage differ in their competence to respond to different inducing signals. Presumptive opV placode ectoderm can respond to Pax2- and Phox2a-inducing signals, but not to Hoxb5-inducing signals. We previously showed that 3-somite stage nodose placode ectoderm is not competent to respond to Pax3-inducing signals in the opV region, while otic placode ectoderm loses this competence after the 3-somite stage (Baker et al., 1999). Recent data suggest that pre-placodal ectoderm, encompassing the whole placodeforming region, is already demarcated by $c$ Six 4 expression in neurula-stage (Hamburger-Hamilton stage 6) chick embryos (Esteve and Bovolenta, 1999; Hamburger and Hamilton, 1951). Hence, it is likely that induction of different placodes is already underway by the 3 -somite stage, changing the competence of different regions to respond to different signals.

\section{Conclusion}

Our results suggest that Pax3 expression within presumptive opV placode ectoderm correlates at a single cell level both with neuronal specification and with commitment to an opV fate. Pax3-negative cells in presumptive opV ectoderm, in contrast, can be induced to express the epibranchial placode marker Pax2 and to form Phox2a-positive neurons in the nodose ganglion. Neurogenesis in the neurogenic placodes does not seem, therefore, to be a two-step process in which neurons are induced and subsequently specified to a particular fate. Instead, neuronal differentiation and neuronal subtype identity seem to be coupled in a one-step induction process. This is similar to observations in other systems undergoing neurogenesis, such as the sensory bristles of Drosophila and the autonomic nervous system of vertebrates.

Thanks to Domingos Henrique and Jean-François Brunet for the Pax2 and Phox $2 a$ in situ probes respectively. We are very grateful to Andy Groves and Helen McBride for discussion and helpful comments on the manuscript. This work was supported by NS-36585 and NS-13815 (M. B.-F.) and by American Heart Association Fellowship 1171-F11 (C. V. H. B.).

\section{REFERENCES}

Anderson, D. J. and Jan, Y. N. (1997). The determination of the neuronal phenotype. In Molecular and Cellular Approaches to Neural Development (ed. W. M. Cowan, T. M. Jessell and S. L. Zipursky). Oxford: Oxford University Press.

Armstrong, J. B. and Malacinski, G. M. (1989). Developmental Biology of the Axolotl. Oxford: Oxford University Press.

Baker, C. V. H., Stark, M. R., Marcelle, C. and Bronner-Fraser, M. (1999). Competence, specification and induction of Pax-3 in the trigeminal placode. Development 126, 147-156.

Begbie, J., Brunet, J.-F., Rubenstein, J. L. R. and Graham, A. (1999). Induction of the epibranchial placodes. Development 126, 895-902.

Brunet, J. F. and Ghysen, A. (1999). Deconstructing cell determination: proneural genes and neuronal identity. BioEssays 21, 313-318.

D'Amico-Martel, A. and Noden, D. M. (1983). Contributions of placodal and neural crest cells to avian cranial peripheral ganglia. Am. J. Anat. 166, 445-468.

Dahl, E., Koseki, H. and Balling, R. (1997). Pax genes and organogenesis. BioEssays 19, 755-765. 
Dambly-Chaudière, C., Jamet, E., Burri, M., Bopp, D., Basler, K., Hafen, E., Dumont, N., Spielmann, P., Ghysen, A. and Noll, M. (1992). The paired box gene pox neuro: a determinant of poly-innervated sense organs in Drosophila. Cell 69, $159-172$.

Davies, A. M. (1998). Developmental changes in the neurotrophic factor survival requirements of peripheral nervous system neurons. Prog. Brain Res. 117, 47-56.

Esteve, P. and Bovolenta, P. (1999). cSix4, a member of the six gene family of transcription factors, is expressed during placode and somite development. Mech. Dev. 85, 161-165.

Favor, J., Sandulache, R., Neuhauser-Klaus, A., Pretsch, W., Chatterjee, B., Senft, E., Wurst, W., Blanquet, V., Grimes, P., Sporle, R. and Schughart, K. (1996). The mouse Pax2 ${ }^{1 \mathrm{Neu}}$ mutation is identical to a human PAX2 mutation in a family with renal-coloboma syndrome and results in developmental defects of the brain, ear, eye, and kidney. Proc. Natl. Acad. Sci. USA 93, 13870-13875.

Fode, C., Gradwohl, G., Morin, X., Dierich, A., LeMeur, M., Goridis, C. and Guillemot, F. (1998). The bHLH protein NEUROGENIN 2 is a determination factor for epibranchial placode-derived sensory neurons. Neuron 20, 483-94.

Gould, A., Itasaki, N. and Krumlauf, R. (1998). Initiation of rhombomeric Hoxb4 expression requires induction by somites and a retinoid pathway. Neuron 21, 3951.

Groves, A. K., George, K. M., Tissier-Seta, J. P., Engel, J. D., Brunet, J. F. and Anderson, D. J. (1995). Differential regulation of transcription factor gene expression and phenotypic markers in developing sympathetic neurons. Development 121, 887-901.

Hamburger, V. and Hamilton, H. L. (1951). A series of normal stages in the development of the chick embryo. J. Morphol. 88, 49-92.

Henrique, D., Adam, J., Myat, A., Chitnis, A., Lewis, J. and Ish-Horowicz, D. (1995). Expression of a Delta homologue in prospective neurons in the chick. Nature 375, 787-790.

Herbrand, H., Guthrie, S., Hadrys, T., Hoffmann, S., Arnold, H.-H., RinkwitzBrandt, S. and Bober, E. (1998). Two regulatory genes, $c N k \times 5-1$ and $c$ Pax2, show different responses to local signals during otic placode and vesicle formation in the chick embryo. Development 125, 645-654.

Hill, R. E., Favor, J., Hogan, B. L. M., Ton, C. T., Saunders, G. F., Hanson, I. M., Prosser, J., Jordan, T., Hastie, N. D. and van Heyningen, V. (1991). Mouse Small eye results from mutations in a paired-like homeobox-containing gene. Nature 354, 522-525.

Hiscock, J. and Straznicky, C. (1986). The development of the neurons of the glossopharyngeal (IX) and vagal (X) sensory ganglia in chick embryos. Histol. Histopathol. 1, 129-137.

Hogan, B. L. M., Horsburgh, G., Cohen, J., Hetherington, C. M., Fisher, G. and Lyon, M. F. (1986). Small eyes (Sey): a homozygous lethal mutation on chromosome 2 which affects the differentiation of both lens and nasal placodes in the mouse. J. Embryol. Exp. Morph. 97, 95-110.

Itasaki, N., Sharpe, J., Morrison, A. and Krumlauf, R. (1996). Reprogramming Hox expression in the vertebrate hindbrain: influence of paraxial mesoderm and rhombomere transposition. Neuron 16, 487-500.

Jacobson, A. G. (1963a). The determination and positioning of the nose, lens and ear. I. Interactions within the ectoderm, and between the ectoderm and underlying tissues. J. Exp. Zool. 154, 273-283.

Jacobson, A. G. (1963b). The determination and positioning of the nose, lens and ear. II. The role of the endoderm. J. Exp. Zool. 154, 285-291.

Jacobson, A. G. (1963c). The determination and positioning of the nose, lens and ear. III. Effects of reversing the antero-posterior axis of epidermis, neural plate and neural fold. J. Exp. Zool. 154, 293-303.

Koblar, S. A., Murphy, M., Barrett, G. L., Underhill, A., Gros, P. and Bartlett, P. F. (1999). Pax-3 regulates neurogenesis in neural crest-derived precursor cells. $J$. Neurosci. Res. 56, 518-530.

Kuratani, S. C. and Wall, N. A. (1992). Expression of Hox 2.1 protein in restricted populations of neural crest cells and pharyngeal ectoderm. Dev. Dyn. 195, 15-28.

Le Douarin, N. M., Fontaine-Perus, J. and Couly, G. (1986). Cephalic ectodermal placodes and neurogenesis. Trends Neurosci. 9, 175-180.

Lee, V. M., Carden, M. J., Schlaepfer, W. W. and Trojanowski, J. Q. (1987). Monoclonal antibodies distinguish several differentially phosphorylated states of the two largest rat neurofilament subunits (NF-H and NF-M) and demonstrate their existence in the normal nervous system of adult rats. J. Neurosci. 7, 3474-3488.

Lindsay, R. M. (1996). Role of neurotrophins and trk receptors in the development and maintenance of sensory neurons: an overview. Phil. Trans. R. Soc. Lond. B 351, 365-373.

Lo, L., Morin, X., Brunet, J. F. and Anderson, D. J. (1999). Specification of neurotransmitter identity by Phox 2 proteins in neural crest stem cells. Neuron 22, 693705 .

Lo, L., Tiveron, M. C. and Anderson, D. J. (1998). MASH1 activates expression of the paired homeodomain transcription factor Phox $2 \mathrm{a}$, and couples pan-neuronal and subtype-specific components of autonomic neuronal identity. Development 125, 609-620.

Mansouri, A., Hallonet, M. and Gruss, P. (1996). Pax genes and their roles in cell differentiation and development. Curr. Opin. Cell Biol. 8, 851-857.

Miller, D. M., Shen, M. M., Shamu, C. E., Burglin, T. R., Ruvkun, G., Dubois, M.
L., Ghee, M. and Wilson, L. (1992). C. elegans unc-4 gene encodes a homeodomain protein that determines the pattern of synaptic input to specific motor neurons. Nature 355, 841-845.

Moody, S. A., Quigg, M. S. and Frankfurter, A. (1989). Development of the peripheral trigeminal system in the chick revealed by an isotype-specific anti-betatubulin monoclonal antibody. J. Comp. Neurol. 279, 567-580.

Morin, X., Cremer, H., Hirsch, M.-R., Kapur, R. P., Goridis, C. and Brunet, J.-F (1997). Defects in sensory and autonomic ganglia and absence of locus coeruleus in mice deficient for the homeobox gene Phox $2 a$. Neuron 18, 411-423.

Perez, S. E., Rebelo, S. and Anderson, D. J. (1999). Early specification of sensory neuron fate revealed by expression and function of neurogenins in the chick embryo. Development 126, 1715-1728.

Perron, M., Opdecamp, K., Butler, K., Harris, W. A. and Bellefroid, E. J. (1999) $X$-ngnr-1 and Xath3 promote ectopic expression of sensory neuron markers in the neurula ectoderm and have distinct inducing properties in the retina. Proc. Natl. Acad. Sci. USA 96, 14996-15001.

Rancourt, D. E., Tsuzuki, T. and Capecchi, M. R. (1995). Genetic interaction between hoxb-5 and hoxb-6 is revealed by nonallelic noncomplementation. Genes Dev. 9, 108-122.

Reyer, R. W. (1962). Differentiation and growth of the embryonic nose, lens and corneal anlagen implanted into the larval eye or dorsal fin in Amblystoma punctatum. J. Exp. Zool. 151, 123-153.

Sechrist, J., Nieto, M. A., Zamanian, R. T. and Bronner-Fraser, M. (1995). Regulative response of the cranial neural tube after neural fold ablation: spatiotemporal nature of neural crest regeneration and up-regulation of Slug. Development 121, 4103-4115.

Serbedzija, G. N. and McMahon, A. P. (1997). Analysis of neural crest cell migration in Splotch mice using a neural crest-specific LacZ reporter. Dev. Biol. 185, 139-147.

Shah, N. M., Groves, A. K. and Anderson, D. J. (1996). Alternative neural crest cell fates are instructively promoted by TGF $\beta$ superfamily members. Cell $\mathbf{8 5}, 331-343$.

Sommer, L., Shah, N., Rao, M. and Anderson, D. J. (1995). The cellular function of MASH1 in autonomic neurogenesis. Neuron 15, 1245-1258.

Stark, M. R., Sechrist, J., Bronner-Fraser, M. and Marcelle, C. (1997). Neural tube-ectoderm interactions are required for trigeminal placode formation. Development 124, 4287-95

Stone, L. S. (1924). Experiments on the transplantation of placodes of the cranial ganglia in the amphibian embryo. I. Heterotopic transplantations of the ophthalmic placode upon the head of Amblystoma punctatum. J. Comp. Neurol. 38, 73-105.

Stone, L. S. (1928). Experiments on the transplantation of placodes of the cranial ganglia in the amphibian embryo. II. Heterotopic transplantations of the ophthalmic placode upon the head and body of Amblystoma punctatum. J. Comp. Neurol. 47, 91-116.

Stone, L. S. (1929). Experiments on the transplantation of placodes of the cranial ganglia in the amphibian embryo. IV. Heterotopic transplantations of the postauditory placodal material upon the head and body of Amblystoma punctatum. J. Comp. Neurol. 48, 311-330.

Strähle, U., Blader, P., Adam, J. and Ingham, P. W. (1994). A simple and efficient procedure for non-isotopic in situ hybridization to sectioned material. Trends Genet. 10, 75-76.

Székely, G. (1959). Functional specificity of cranial sensory neuroblasts in Urodela. Acta Biol. Acad. Sci. Hung. 10, 107-115.

Tanaka, H., Kinutani, M., Agata, A., Takashima, Y. and Obata, K. (1990). Pathfinding during spinal tract formation in the chick-quail chimera analysed by species-specific monoclonal antibodies. Development 110, 565-571.

Torres, M., Gomez-Pardo, E. and Gruss, P. (1996). Pax2 contributes to inner ear patterning and optic nerve trajectory. Development 122, 3381-3391.

Trainor, P. and Krumlauf, R. (2000). Plasticity in mouse neural crest cells reveals a new patterning role for cranial mesoderm. Nat. Cell Biol. 2, 96-102.

Tremblay, P., Kessel, M. and Gruss, P. (1995). A transgenic neuroanatomical marker identifies cranial neural crest deficiencies associated with the Pax3 mutant Splotch. Dev. Biol. 171, 317-329.

Valarché, I., Tissier-Seta, J. P., Hirsch, M. R., Martinez, S., Goridis, C. and Brunet, J. F. (1993). The mouse homeodomain protein Phox2 regulates Ncam promoter activity in concert with Cux/CDP and is a putative determinant of neurotransmitter phenotype. Development 119, 881-896.

Vervoort, M., Zink, D., Pujol, N., Victoir, K., Dumont, N., Ghysen, A. and Dambly-Chaudière, C. (1995). Genetic determinants of sense organ identity in Drosophila: regulatory interactions between cut and poxn. Development 121,31113120 .

Vogel, K. S. and Davies, A. M. (1993). Heterotopic transplantation of presumptive placodal ectoderm changes the fate of sensory neuron precursors. Development 119, 263-276.

Wall, N. A., Jones, C. M., Hogan, B. L. M. and Wright, C. V. E. (1992). Expression and modification of Hox 2.1 protein in mouse embryos. Mech. Dev. 37, 111-120.

Webb, J. F. and Noden, D. M. (1993). Ectodermal placodes: contributions to the development of the vertebrate head. Amer. Zool. 33, 434-447.

Zhao, C. and Emmons, S. W. (1995). A transcription factor controlling development of peripheral sense organs in C. elegans. Nature 373, 74-78. 